\title{
Dependency of repair of radiation-induced damage on p53 status and efficient inhibition of the repair with wortmannin treatment in vivo, with reference to the effect on intratumor quiescent cell population
}

\author{
SHIN-ICHIRO MASUNAGA ${ }^{1}$, AKIHISA TAKAHASHI ${ }^{2}$, KEN OHNISHI $^{2}$, KENJI NAGATA ${ }^{1}$, \\ MINORU SUZUKI $^{1}$, GENRO KASHINO ${ }^{1}$, YUKO KINASHI ${ }^{2}$, TAKEO OHNISHI ${ }^{2}$ and KOJI ONO ${ }^{1}$ \\ ${ }^{1}$ Particle Radiation Oncology Research Center and ${ }^{2}$ Division of Radiation Safety, Research Reactor Institute, \\ Kyoto University, 2-1010 Asashiro-nishi, Kumatori-cho, Sennan-gun, Osaka 590-0494; ${ }^{2}$ Department of Biology, \\ Nara Medical University, 840 Shijo-cho, Kashihara, Nara 634-8521, Japan
}

Received May 10, 2007; Accepted August 8, 2007

\begin{abstract}
To examine the dependency of p53 status and the effect of wortmannin on the repair of radiation-induced damage, referring to the response of intratumor quiescent (Q) cells. Human head and neck squamous cell carcinoma cells transfected with mutant TP53 (SAS/mp53) or with neo vector (SAS/neo) were injected subcutaneously into left hind legs of nude mice. Tumor-bearing mice received 5-bromo2'-deoxyuridine (BrdU) continuously to label all intratumor proliferating $(\mathrm{P})$ cells. They received high dose-rate $\gamma$-ray irradiation (HDRI) immediately followed by caffeine or wortmannin administration, or reduced dose-rate $\gamma$-ray irradiation simultaneously with caffeine or wortmannin administration. Nine hours after the start of irradiation, the tumor cells were isolated and incubated with a cytokinesis blocker, and the micronucleus (MN) frequency in cells without BrdU labeling ( $Q$ cells) was determined using immunofluorescence staining for BrdU. SAS/neo tumor cells, especially intratumor Q cell populations, showed a marked reduction in sensitivity due to the repair of radiationinduced damage, compared with the total or Q cell populations within SAS/mp53 tumors that showed little repair capacity. In both total and Q cell populations within SAS/neo tumors, wortmannin efficiently suppressed the reduction in sensitivity caused by leaving an interval between HDRI and the assay
\end{abstract}

Correspondence to: Dr Shin-ichiro Masunaga, Particle Radiation Oncology Research Center, Research Reactor Institute, Kyoto University, 2-1010 Asashiro-nishi, Kumatori-cho, Sennan-gun, Osaka 590-0494, Japan

E-mail: smasuna@rri.kyoto-u.ac.jp

Key words: p53 status, dose-rate effect, quiescent cell, wortmannin, caffeine and decreasing the irradiation dose rate. The repair of radiationinduced damage was thought to be a p53-dependent event. From the viewpoint of tumor control, including intratumor Q-cell control, wortmannin treatment in combination with $\gamma$-ray irradiation is thought to be useful for suppressing the repair of radiation-induced damage.

\section{Introduction}

Genomic instability is a major force driving human cancer development. The p53 tumor suppressor gene serves a critical role in maintaining genomic stability during the cell cycle checkpoint in not only G1 but also the G2/M transition, as an effector of DNA repair and apoptosis. Wild-type p53 is liable to activate apoptosis in response to DNA damage $(1,2)$. These actions of p53 are potentially critical in determining the effectiveness of ionizing radiation. Actually, mutations in the p53 tumor suppressor gene have been shown to have an impact on the clinical course of several cancers: patients with cancers harboring p53 mutations often have a worse prognosis than those with tumors harboring wild-type p53 $(1,2)$. Thus, the genetic and functional status of the p53 gene is thought to be an important factor in guiding therapeutic strategies for cancer patients.

Many cells in solid tumors are quiescent in situ but are still clonogenic (3). These quiescent (Q) tumor cell populations have been thought to be more resistant to irradiation because of their much larger hypoxic fractions and greater potentially lethal damage repair (PLDR) capacities than proliferating (P) tumor cells, mainly based on the characteristics of plateau-phase cultured cells in vitro (3). Employing our method for selectively detecting the response of intratumor $\mathrm{Q}$ cell populations in vivo, we have already shown that all these characteristics could be applied to Q cells in solid tumors in vivo under conventional high dose-rate irradiation (HDRI) conditions (4). However, clinically, reduced doserate irradiation (RDRI) was found to spare normal tissue from radiation-induced damage resulting in a greater therapeutic 
gain, because the therapeutic ratio is equal to the ratio of tumor control to normal tissue complications (5).

Two major pathways for the repair of potentially lethal DNA double-stranded breaks (dsbs) exist in mammalian cells. The non-homologous end-joining (NHEJ) pathway is imprecise, error-prone, and mutagenic, and mutant cell lines lacking key components of this pathway all exhibit impaired kinetics of DNA dsb repair and exquisite radiosensitivity $(1,6)$. Homologous recombination (HR) is a more precise (error-free) repair mechanism and is more important for the repair of dsbs in late-S and G2 when a sister chromatid is available for the recombination reaction. Cell lines with defects in HR also exhibit increased radiosensitivity and decreased fidelity of repair $(1,7)$. Wortmannin is known to have the potential to hinder NHEJ repair by inhibiting a catalytic subunit of DNA-dependent protein kinase (8). Caffeine is known to inhibit HR by targeting ataxia telangiectasia mutated protein kinase (ATM) and ATM- and Rad3-related protein kinase (ATR) (9).

Here, the usefulness of wortmannin or caffeine treatment right after HDRI or concurrently with RDRI with low linear energy transfer (LET) radiation $\gamma$-rays, including the dependency on p53 status of tumor cells using tumor cell lines with identical genetic backgrounds except for p53 status, was evaluated in terms of the extent of the repair of radiation-induced damage, using our method for selectively detecting the responses of the total $(\mathrm{P}+\mathrm{Q})$ and $\mathrm{Q}$ cell populations in solid tumors (4).

\section{Materials and methods}

Cells, tumors and mice. The human head and neck squamous cell carcinoma cell line SAS (provided by JCRB, Tokyo Japan) was cultured at $37^{\circ} \mathrm{C}$ in Dulbeco's modified Eagle's medium containing $20 \mathrm{mM}$ 2-[4-(2-hydroxyethyl)-1-piperazinyl]ethanesulfonic acid (HEPES) and $12.5 \%$ fetal bovine serum (DMEM) in a conventional humified $5 \% \mathrm{CO}_{2}$ incubator. SAS cells show the phenotype of wild-type p53 in radiationand heat-induced signal transduction $(10,11)$, although they have a point mutation at codon 336 of exon 10 in the p53 gene (12). The plasmids pC53-248, which contains an mp53 gene (codon 248, from Arg to Trp) producing a dominant negative mp53 protein, and pCMV-Neo-Bam, which contains a neo-resistant marker, were provided by B. Vogelstein (Johns Hopkins Oncology Center, Baltimore, MD). These plasmids were linearized with HindIII. Confluent SAS cells, $\sim 2 \times 10^{6}$ cells in a $75-\mathrm{cm}^{2}$ flask, were trypsinized, and the resulting cell suspension in phosphate-buffered saline (PBS) $(1 \mathrm{ml})$ was transferred into an electroporation chamber. Cells were supplemented with linearized DNA (10 $\mu \mathrm{g} / 10 \mu \mathrm{l}$ of pC53-248 or pCMV-Neo-Bam), and electroporated three times at $600 \mathrm{~V}$. After standing for $30 \mathrm{~min}$ at room temperature, cells were plated onto dishes $10 \mathrm{~cm}$ in diameter in DMEM and incubated at $37^{\circ} \mathrm{C}$. Forty-eight hours later, cells were treated with G418 (geneticin, $200 \mu \mathrm{g} / \mathrm{ml}$, Sigma Chemical Co. St. Louis, MO), an agent for the selection of transfected clones, and then incubated at $37^{\circ} \mathrm{C}$ for 14 days to allow colonies to form. Colonies resistant to G418 were isolated with cloning cylinders. Through these manipulations, two stable transfectants, SAS/mp53 and SAS/neo, were established. SAS/neo cells have a functional wild-type p53 protein, while SAS/mp53 cells express a dominant negative $\mathrm{p} 53$ protein. The procedure used for transfection is described in detail elsewhere (13).

Cells were collected from exponentially growing cultures, and $\sim 5.0 \times 10^{5}$ cells were inoculated subcutaneously into left hind legs of 6- to 7-week-old syngeneic female Balb/cA nude mice. Three weeks after the inoculation, a tumor with a diameter of $\sim 7 \mathrm{~mm}$ could be observed at each implanted site, whichever stable transfectant was used.

Labeling with 5-bromo-2'-deoxyuridine (BrdU). Two weeks after the tumor cell inoculation, mini-osmotic pumps (Alzet model 2001, DURECT Corp., Cupertino, CA) containing BrdU dissolved in physiological saline $(250 \mathrm{mg} / \mathrm{ml})$ were implanted subcutaneously to label all $\mathrm{P}$ cells for 7 days. Administration of BrdU did not change the tumor growth rate. The tumors were approximately $7 \mathrm{~mm}$ in diameter on treatment. The labeling index (LI) after continuous labeling with BrdU was $48.4 \%$ (41.7-55.1\%) [mean (95\% confidence limit)] and $43.2 \%$ (37.0-49.4\%) for SAS/neo and SAS/mp53 tumor cells, respectively, and reached a plateau level at these stages. Therefore, we regarded tumor cells not incorporating BrdU after continuous labeling as Q cells.

Treatment. After the labeling with BrdU, tumor-bearing mice received $\gamma$-ray irradiation. $\gamma$-ray irradiation was performed with a cobalt-60 $\gamma$-ray irradiator at a dose rate of $2.75 \mathrm{~Gy} / \mathrm{min}$ as HDRI with tumor-bearing mice held in a specially constructed device with the tail firmly fixed with an adhesive tape. RDRI was performed at a dose rate of $0.035 \mathrm{~Gy} / \mathrm{min}$ by maintaining an appropriate distance between the cobalt- 60 radiation source and the irradiated tumor-bearing mouse fixed within the specially constructed device.

In addition, wortmannin [6 mg/kg) or caffeine $(68.5 \mathrm{mg} / \mathrm{kg}$ $=1 / 2$ LD50 (mean lethal dose)] dissolved in physiological saline $(8,9)$ was also administered intraperitoneally immediately after HDRI or immediately before the start of RDRI. Each irradiation group also included mice that were not pretreated with BrdU.

Immunofluorescence staining of BrdU-labeled cells and micronucleus (MN) assay. Nine hours after HDRI or $9 \mathrm{~h}$ after the starting point of RDRI, tumors were excised from the mice given BrdU, minced, and trypsinized. Tumor cell suspensions thus obtained were incubated for $72 \mathrm{~h}$ in tissue culture dishes containing complete medium and $1.0 \mu \mathrm{g} / \mathrm{ml}$ of cytochalasin-B to inhibit cytokinesis while allowing nuclear division, and the cultures were then trypsinized and cell suspensions were fixed. After the centrifugation of fixed cell suspensions, the cell pellet was resuspended with cold Carnoy's fixative (ethanol: acetic acid $=3: 1$ in volume). The suspension was then placed on a glass microscope slide and the sample was dried at room temperature. The slides were treated with $2 \mathrm{M}$ hydrochloric acid for $60 \mathrm{~min}$ at room temperature to dissociate the histones and partially denature the DNA. The slides were then immersed in borax-borate buffer $(\mathrm{pH} \mathrm{8.5)}$ to neutralize the acid. BrdU-labeled tumor cells were detected by indirect immunofluorescence staining using monoclonal anti-BrdU antibody (Becton Dickinson, San Jose, CA) and fluorescein isothiocyanate (FITC)-conjugated antimouse IgG antibody 
Table I. Plating efficiencies and micronucleus frequencies at $0 \mathrm{~Gy}$.

\begin{tabular}{|c|c|c|}
\hline Treatment & SAS/neo & $\mathrm{SAS} / \mathrm{mp} 53$ \\
\hline \multicolumn{3}{|c|}{ Plating efficiency (\%) } \\
\hline None & $48.4 \quad(39.3-57.5)^{\mathrm{a}}$ & $28.5 \quad(22.4-34.6)$ \\
\hline Caffeine & $28.3 \quad(24.3-32.3)$ & $17.9(14.9-20.9)$ \\
\hline Wortmannin & $28.6 \quad(25.3-31.9)$ & $18.1 \quad(15.6-20.6)$ \\
\hline \multicolumn{3}{|c|}{ Micronucleus frequency } \\
\hline \multicolumn{3}{|c|}{ Total tumor cells } \\
\hline None & $0.038(0.032-0.044)$ & $0.072(0.064-0.080)$ \\
\hline Caffeine & $0.088(0.079-0.097)$ & $0.137(0.127-0.147)$ \\
\hline Wortmannin & $0.086(0.078-0.094)$ & $0.136(0.120-0.152)$ \\
\hline \multicolumn{3}{|c|}{ Quiescent tumor cells } \\
\hline None & $0.056(0.049-0.063)$ & $0.10 \quad(0.09-0.011)$ \\
\hline Caffeine & $0.114(0.105-0.122)$ & $0.177(0.164-0.190)$ \\
\hline Wortmannin & $0.116(0.103-0.129)$ & $0.181(0.161-0.201)$ \\
\hline
\end{tabular}

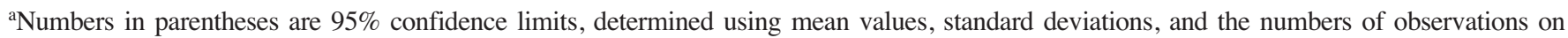
which the means and standard deviations were based.

(Sigma, St. Louis, MO). To observe the double staining of tumor cells with green-emitting FITC and red-emitting propidium iodide (PI), cells on the slides were treated with PI $[2 \mu \mathrm{g} / \mathrm{ml}$ in phosphate-buffered saline (PBS)] and monitored under a fluorescence microscope.

The MN frequency in cells not labeled with BrdU could be examined by counting the micronuclei in the binuclear cells that showed only red fluorescence. The MN frequency was defined as the ratio of the number of micronuclei in the binuclear cells to the total number of binuclear cells observed (4).

The ratios obtained in tumors not pretreated with BrdU indicated the MN frequency at all phases in the total $(\mathrm{P}+\mathrm{Q})$ tumor cell population. More than 400 binuclear cells were counted to determine the $\mathrm{MN}$ frequency.

Clonogenic cell survival assay. The clonogenic cell survival assay was also performed in the mice given no BrdU using an in vivo-in vitro assay method. Tumors were disaggregated by stirring for $20 \mathrm{~min}$ at $37^{\circ} \mathrm{C}$ in PBS containing $0.05 \%$ trypsin and $0.02 \%$ ethylenediamine tetraacetic acid. The cell yield was $1.5(1.2-1.8) \times 10^{7} / \mathrm{g}$ and $3.4(2.6-4.2) \times 10^{6} / \mathrm{g}$ for SAS/neo and SAS/mp53 tumors, respectively. Appropriate numbers of viable tumor cells from the single cell suspension were plated on 60 or $100 \mathrm{~mm}$ tissue culture dishes, and 16 days later, colonies were fixed with ethanol, stained with Giemsa, and counted. For the tumors that received no irradiation, the plating efficiencies for the total tumor cell populations and the MN frequencies for the total and Q cell populations are shown in Table I.

To confirm the stability of transfectants SAS/neo and SAS/mp53, parts of tumor cell suspensions obtained after $\gamma$-ray irradiation to solid tumors and tumor cells from part of the colonies grown using the in vivo-in vitro assay method were subjected to Western blotting for p53 and Bax proteins as described in Ota et al (10). Not only the protein level but also the function of p53 can be detected because the bax gene is one of p53's targets. As a result, it was certified that the p53 status of each transfectant was not changed by these experimental procedures. Three mice were used to assess each set of conditions and each experiment was repeated twice. To examine the differences between pairs of values, Student's t-test was used when variances of the two groups could be assumed to be equal; otherwise the Welch t-test was used. P-values are from two-sided tests.

\section{Results}

Table I shows the plating efficiencies for the total tumor cell population and the $\mathrm{MN}$ frequencies without $\gamma$-ray radiation for the total and Q cell populations in each tumor. Overall, SAS/mp53 tumor cells showed significantly lower plating efficiency in the total cell populations and significantly higher MN frequencies in both the total and Q cell populations $(\mathrm{P}<0.05)$. Further, $\mathrm{Q}$ cells showed significantly higher $\mathrm{MN}$ frequencies than the total cell population under each set of conditions in each tumor $(\mathrm{P}<0.05)$. Wortmannin and caffeine induced significantly lower plating efficiencies and significantly higher MN frequencies in both the total and Q cell populations within each tumor than absolutely no treatment $(\mathrm{P}<0.05)$. Furthermore, the values of the plating efficiency and the MN frequency for both drugs were almost the same. Thus, it follows that the dose of each drug employed here had an almost iso-effect in the absence of $\gamma$-ray irradiation.

The clonogenic cell survival curves for total tumor cell populations and the normalized $\mathrm{MN}$ frequencies of total and Q cell populations after $\gamma$-ray irradiation with HDRI or RDRI are shown in Fig. 1A and B, respectively. For baseline 

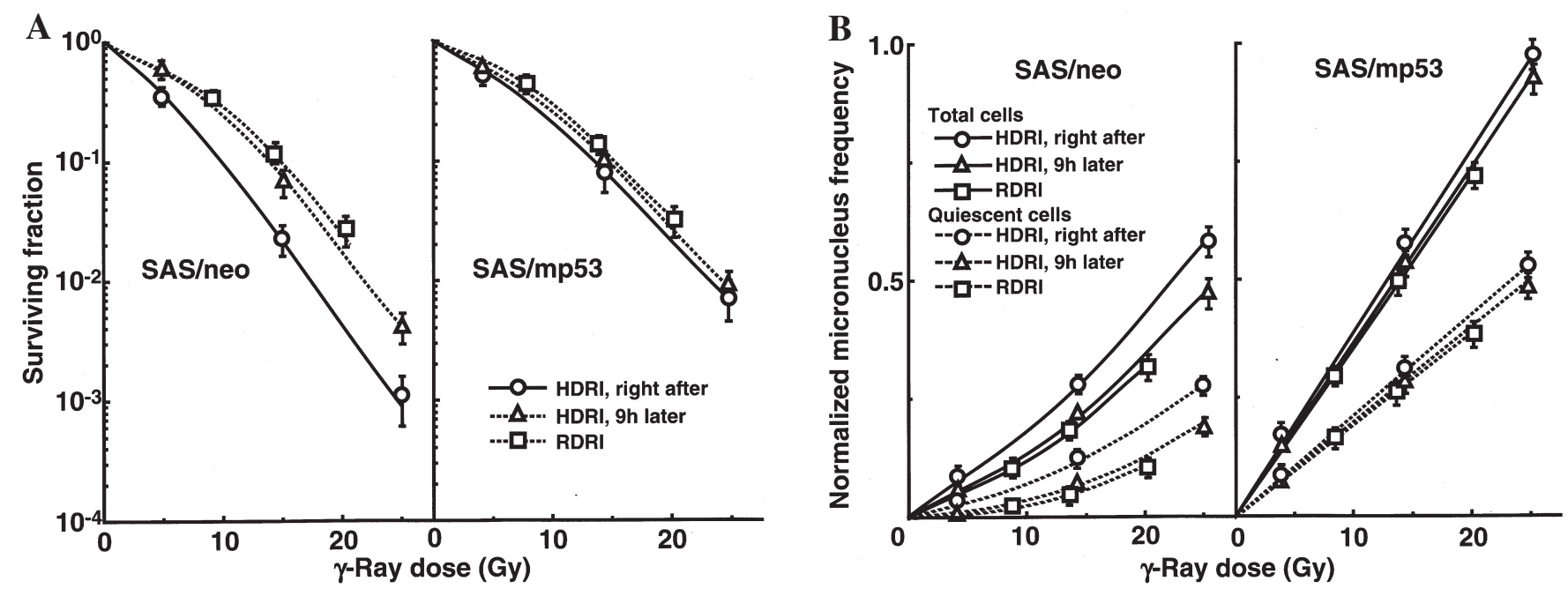

Figure 1. The clonogenic cell survival curves for total tumor cell populations and the normalized micronucleus frequencies for total and quiescent cell populations immediately and $9 \mathrm{~h}$ after $\gamma$-ray irradiation with high dose-rate irradiation (HDRI) and immediately after $\gamma$-ray irradiation with reduced dose-rate irradiation (RDRI) are shown in A and B, respectively. The left and right panels show SAS/neo and SAS/mp53 tumor cells, respectively. Bars represent $95 \%$ confidence limits.
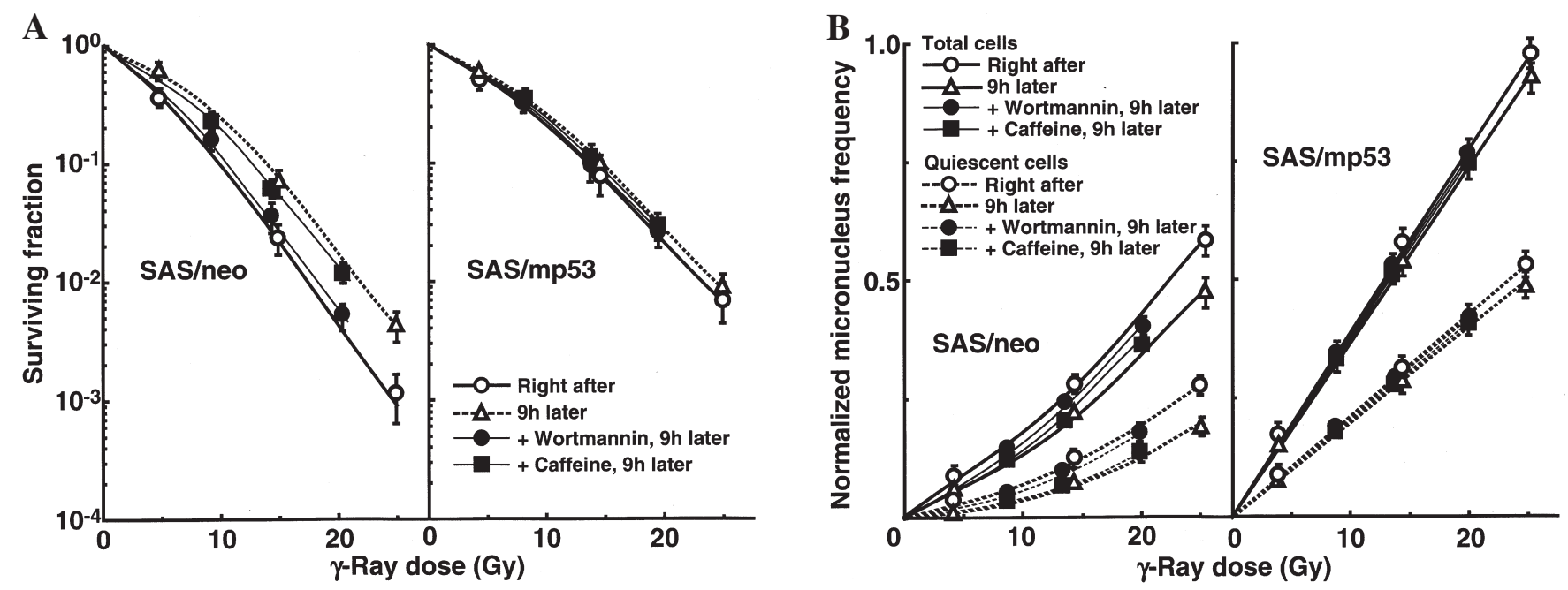

Figure 2. The clonogenic cell survival curves for total tumor cell populations and the normalized micronucleus frequencies for total and quiescent cell populations immediately after and $9 \mathrm{~h}$ after $\gamma$-ray irradiation with high dose-rate irradiation (HDRI) immediately followed by the administration of caffeine, that of wortmannin, or no treatment are shown in A and B, respectively. The left and right panels show SAS/neo and SAS/mp53 tumor cells, respectively. Bars represent $95 \%$ confidence limits.

correction, we used the normalized MN frequency to exclude the effect of wortmannin or caffeine in non-irradiated control tumors. The normalized MN frequency was the MN frequency in the irradiated tumors minus that in the nonirradiated tumors. On the whole, SAS/mp53 tumor cells and Q tumor cells were more radioresistant than SAS/neo tumor cells and the total tumor cell population, respectively. The increase in the surviving fraction (SF) with the $9 \mathrm{~h}$ delayed assay, that is, PLDR, and the increase in the SF after RDRI were observed more clearly in SAS/neo than in SAS/mp53. The decrease in the normalized MN frequency with the $9 \mathrm{~h}$ delayed assay and the decrease in the normalized MN frequency after RDRI were more obvious in SAS/neo and Q cells than in SAS/mp53 and the total cell population, respectively.
The clonogenic cell survival curves for total tumor cell populations and the normalized $\mathrm{MN}$ frequencies of total and Q cell populations after $\gamma$-ray irradiation with HDRI immediately followed by the administration of wortmannin or caffeine, or no treatment are shown in Fig. 2A and B, respectively. PLDR was suppressed in combination with wortmannin much more clearly than with caffeine in both the total and Q cell populations.

The clonogenic cell survival curves for total tumor cell populations and the normalized MN frequencies of total and Q cell populations after $\gamma$-ray irradiation with RDRI in simultaneous combination with wortmannin or caffeine, or no treatment are also shown in Fig. 3A and B, respectively. The increase in the SF and the decrease in the normalized MN frequency after RDRI were repressed in combination 

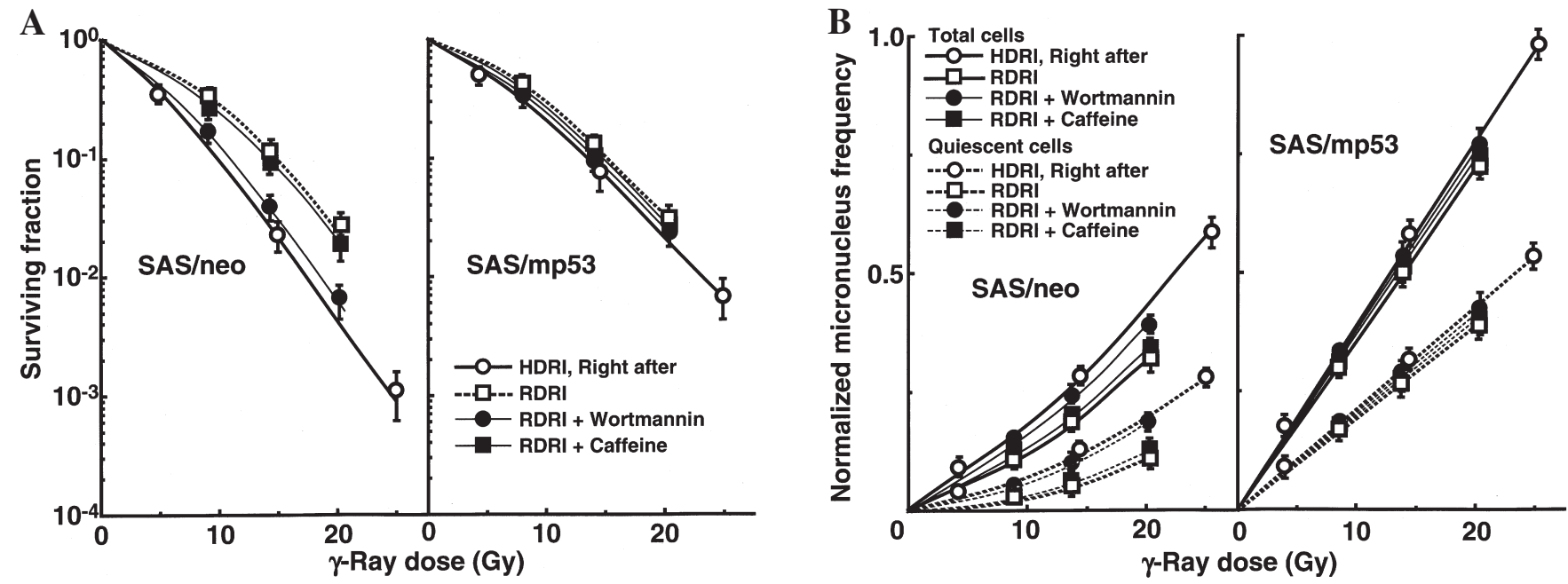

Figure 3. The clonogenic cell survival curves for total tumor cell populations and the normalized micronucleus frequencies for total and quiescent cell populations immediately after $\gamma$-ray irradiation with high dose-rate irradiation (HDRI) and after $\gamma$-ray irradiation with reduced dose-rate irradiation (RDRI) in simultaneous combination with the administration of caffeine or wortmannin, or no treatment are shown in A and B, respectively. The left and right panels show SAS/neo and SAS/mp53 tumor cells, respectively. Bars represent 95\% confidence limits.

Table II. Dose-modifying factors by repair. ${ }^{\mathrm{a}}$

Surviving fraction $=0.04$

Total tumor cells

Right after HDRI ${ }^{\mathrm{b}}$

$9 \mathrm{~h}$ after HDRI

$\mathrm{WM}^{\mathrm{d}}$ right after HDRI, $9 \mathrm{~h}$ later

$\mathrm{CAF}^{\mathrm{e}}$ right after HDRI, $9 \mathrm{~h}$ later

After RDRI ${ }^{\mathrm{f}}$

With WM during RDRI

With CAF during RDRI
1.0

$1.3(1.2-1.4)^{c}$

$1.05(1.0-1.1)$

$1.25(1.2-1.3)$

$1.4(1.3-1.5)$

$1.05(1.0-1.1)$

$1.35(1.3-1.4)$
1.0

$1.05(1.0-1.1)$

$1.02(1.0-1.05)$

$1.05(1.0-1.1)$

$1.1 \quad(1.0-1.1)$

$1.05(1.0-1.1)$

$1.07(1.05-1.1)$

Normalized micronucleus frequency $=0.1$

Total tumor cells

Right after HDRI

1.0

$1.25(1.3-1.4)$

$1.1 \quad(1.0-1.2)$

$1.2(1.1-1.3)$

$1.3(1.2-1.4)$

$1.1 \quad(1.0-1.2)$

$1.2(1.1-1.4)$

1.0

1.5 (1.4-1.6)

1.1 (1.0-1.2)

$1.35(1.3-1.4)$

1.65 (1.6-1.7)

$1.1 \quad(1.0-1.2)$

1.45 (1.4-1.5)
1.0

1.05 (1.0-1.1)

$1.03(1.0-1.05)$

1.05 (1.0-1.1)

$1.1 \quad(1.0-1.2)$

$1.03(1.0-1.05)$

$1.07(1.05-1.1)$

1.0

1.07 (1.05-1.1)

$1.02(1.0-1.05)$

1.05 (1.0-1.1)

1.15 (1.1-1.2)

$1.02(1.0-1.05)$

1.1 (1.0-1.1)

aThe dose of radiation required to obtain each end-point with each treatment in relation to that required to obtain each end-point immediately after HDRI. ${ }^{b}$ High dose-rate irradiation. ${ }^{c}$ Numbers in parentheses are $95 \%$ confidence limits, determined using mean values, standard deviations, and the numbers of observations on which the means and standard deviations were based. ${ }^{\mathrm{d}}$ Wortmannin. ${ }^{\mathrm{e}} \mathrm{Caffeine} .{ }^{\mathrm{f}}$ Reduced dose-rate irradiation. 
Table III. Dose ratios for quiescent tumor cells relative to the total tumor cell population. ${ }^{\mathrm{a}}$

\begin{tabular}{|c|c|c|}
\hline & SAS/neo & $\mathrm{SAS} / \mathrm{mp} 53$ \\
\hline \multicolumn{3}{|l|}{$\begin{array}{l}\text { Normalized micro- } \\
\text { nucleus frequency }=0.1\end{array}$} \\
\hline Right after HDRI ${ }^{\mathrm{b}}$ & $2.2(2.1-2.3)^{\mathrm{c}}$ & $1.9(1.8-2.0)$ \\
\hline $9 \mathrm{~h}$ after HDRI & $2.35(2.25-2.45)$ & $1.95(1.85-2.05)$ \\
\hline $\mathrm{WM}^{\mathrm{d}}$ right after HDRI, & & \\
\hline $9 \mathrm{~h}$ later & $2.1 \quad(2.0-2.2)$ & $1.85(1.75-1.95)$ \\
\hline $\mathrm{CAFe}^{\mathrm{right}}$ after HDRI, & & \\
\hline $9 \mathrm{~h}$ later & $2.3(2.2-2.4)$ & $1.9(1.8-2.0)$ \\
\hline After RDRI ${ }^{\mathrm{f}}$ & $2.25(2.15-2.35)$ & $1.95(1.85-2.05)$ \\
\hline With WM during RDRI & $2.1 \quad(2.0-2.2)$ & $1.85(1.75-1.95)$ \\
\hline With CAF during RDRI & $2.25(2.15-2.35)$ & $1.9(1.8-2.0)$ \\
\hline
\end{tabular}

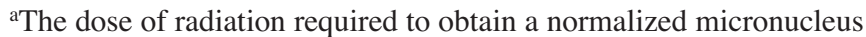
frequency of 0.1 in quiescent tumor cells in relation to that required to obtain a normalized micronucleus frequency of 0.1 in the total tumor cell population. ${ }^{b}$ High dose-rate irradiation. ${ }^{\mathrm{c}}$ Numbers in parentheses are $95 \%$ confidence limits, determined using mean values, standard deviations, and the numbers of observations on which the means and standard deviations were based. ${ }^{\mathrm{d}}$ Wortmannin. ${ }^{\mathrm{e}}$ Caffeine. ${ }^{\mathrm{f}}$ Reduced dose-rate irradiation.

with wortmannin much more clearly than with caffeine in both cell populations. To compare the cell survival curves between these two tumor cells, we calculated the dose ratios for SAS/mp53 tumor cells relative to SAS/neo tumor cells. The factors were calculated by comparing the radiation doses to obtain $\mathrm{SF}=0.04$ in SAS/mp53 tumor cells with the doses required in SAS/neo tumor cells. The values of the dose ratios for $\mathrm{SF}=0.04$ were 1.35 (1.3-1.4), 1.05 (1.0-1.1), 1.3 $(1.25-1.35)$ and $1.1(1.05-1.15)$ when tumors were excised from mice right after HDRI, $9 \mathrm{~h}$ after HDRI, $9 \mathrm{~h}$ after wortmannin administration following HDRI, and $9 \mathrm{~h}$ after caffeine administration following HDRI, respectively. The values were 1.05 (1.0-1.1), 1.3 (1.25-1.35) and 1.05 (1.0-1.1) when tumors were excised from mice right after RDRI, right after RDRI in simultaneous combination with wortmannin, and right after RDRI in simultaneous combination with caffeine, respectively. The values were decreased by the delayed assay after HDRI or a decreasing irradiation dose rate because of the apparent repair of radiation-induced damage in SAS/neo tumor cells, compared with SAS/mp53 tumor cells that showed little repair capacity. However, the clear repair-inhibiting effect of wortmannin kept the values almost constant.

To evaluate the effect of wortmannin and caffeine on the repair of damage induced by HDRI or during RDRI in total and Q cell populations within these two tumors, dosemodifying factors were calculated in both cell populations at various endpoints using the data given in Figs. 1-3 (Table II). Overall, regardless of the cell populations, SAS/mp53 tumor cells showed little repair capacity under any irradiation conditions. In SAS/neo tumor cells, whether $9 \mathrm{~h}$ after HDRI or after RDRI, the repair capacities were significantly greater in $\mathrm{Q}$ cell populations than total cell populations $(\mathrm{P}<0.05)$. Wortmannin treatment right after HDRI significantly inhibited repair of the damage induced by HDRI with a more remarkable inhibition in Q cells than in the total cell population. The simultaneous combination of wortmannin and RDRI significantly inhibited repair of the damage caused by RDRI more efficiently than did the simultaneous combination of caffeine, with a more remarkable inhibition in Q cells than in the total cell population.

Table III shows the dose ratios of Q cells relative to total tumor cell populations; these factors were used to compare the radiation doses necessary to obtain the normalized $\mathrm{MN}$ frequency of 0.1 in $\mathrm{Q}$ cells with the doses required in the total tumor cell populations. All the values of the dose ratios were significantly larger than $1.0(\mathrm{P}<0.05)$. Wortmannin treatment right after HDRI and simultaneously with RDRI, especially in SAS/neo tumors, reduced the widened difference in $\gamma$-ray sensitivity between the total and Q tumor cell populations due to a greater repair capacity in Q cells than in the total cell population at $9 \mathrm{~h}$ after HDRI and after RDRI, respectively.

\section{Discussion}

Potentially lethal damage (PLD) is the component of radiation damage that can be modified by post-irradiation conditions (5). Under ordinary circumstances, PLD causes cell death. Changing cellular growth conditions or the microenvironment of cells influences the expression of PLD or its repair (PLDR), and thereby influences sensitivity to radiation. PLDR is favored by conditions that maintain cells without encouraging or allowing them to divide. Conditions found in solid tumors, regions of which may be far from blood vessels and low in glucose and oxygen, have a low extracellular $\mathrm{pH}$, and show high concentrations of cellular waste products, may prevent cells from proliferating and thereby promote the repair of PLD. Extensive studies on PLDR suggest that DNA dsbs are potentially lethal lesions that can be converted into lethal damage (5). It was recently reported that the conversion of potentially lethal lesions into lethal lesions might be a p53-dependent process and that PLDR was proportional to the percentage of radiation-induced DNA dsbs rejoined in $1 \mathrm{~h}$ in the cell lines with a normal p53 $(14,15)$.

Dose rate is one of the principal factors determining the biologic consequences of a given absorbed dose. As the dose rate is lowered and the exposure time extended, the biologic effect of a given dose is generally reduced. The dose-rate effect, which is very important in radiotherapy, results from the repair of sublethal damage that occurs during a long radiation exposure (5). Incidentally, sublethal damage repair is the operational term for the increase in cell survival that is observed if a given radiation dose is split into two fractions separated by a time interval. Because continuous low doserate irradiation may be considered to be an infinite number of infinitely small fractions, the survival curve under these conditions also would be expected to have no shoulder and to be shallower than for a single acute exposure (5). It was also reported that a normal functioning p53 gene is indispensable for a repair of DNA damage induced under low dose-rate irradiation $(16,17)$. 
A cellular safeguard against genetic destabilization is activation of the p53 tumor suppressor protein, which commonly responds to DNA damage signals by inducing apoptosis or regulating the cell cycle including DNA damage repair $(1,2)$. In fact, as also shown in our previous report $(18)$ and this study, the normalized MN frequencies in SAS/neo tumor cells were lower than those in SAS/mp53 tumor cells under all conditions $(\mathrm{P}<0.05)$, probably resulting from exclusion of a higher number of radiation-induced apoptotic SAS/neo cells than SAS/mp53 cells.

Concerning whether PLDR after HDRI or the repair during RDRI, SAS/neo showed an apparent repair phenomenon in both total and Q cell populations (Fig. 1 and Table II). Notably, Q cells in solid tumors with wild-type p53 exhibited greater capacities of the repair than the total cell population, probably due to the intratumor conditions, that is, hypoxic, nutrition-depleted, and low pH surroundings, where Q cells came into existence (3) (Fig. 1 and Table II). In contrast, no apparent repair was observed in total or Q-cell populations within p53-mutated tumors (Fig. 1 and Table II).

It has been thought that decreasing the dose rate reduces late effects in normal tissue much more than it decreases tumor control. Thus, the 'therapeutic ratio' increases as the dose rate decreases, because the therapeutic ratio is equal to the ratio of tumor control to normal tissue complications. Further, the difference between early and late effects for low dose-rate radiotherapy, as well as improving the therapeutic ratio, allows the delivery of a complete treatment in a short time, allowing the effects of tumor repopulation to be minimized. In other words, decreasing the dose rate increases the therapeutic ratio, limited by tumor cell repopulation (5). This is the primary rationale for low dose-rate radiotherapy. However, this rationale does not take into account the response of $\mathrm{Q}$ tumor cells. The current study showed that lowering the dose rate decreases the effect on $\mathrm{Q}$ cells more markedly than it reduces the effect on the total cell population (Table II). Therefore, considering the Q-cell response, it follows that the therapeutic ratio does not always increase when the dose rate is reduced. Thus, there is a need to overcome this disadvantageous phenomenon.

Wortmannin was originally described as a potent inhibitor of phosphoinositide 3-kinases (PI-3Ks), which are lipid kinases involved in insulin and other mitogenic signaling pathways. The kinase domain of PI-3K shares homology with ataxia telangiectasia mutated (ATM) protein, which is a protein kinase that functions in DNA damage-responsive signaling pathways by phosphorylating target proteins. ATM is related to several other protein kinases involved in the regulation of eukaryotic cell cycle progression and DNA damage-triggered responses $(7,8)$. These proteins all contain a carboxy-terminal kinase domain that shares significant sequence homology with the kinase domains of mammalian and yeast PI-3Ks. In this family of PI-3K-related kinases, there is a catalytic subunit of the DNA-dependent protein kinase (DNA-PKcs) that functions in the NHEJ DNA repair pathway. Thus, wortmannin shows the potential to inhibit the catalytic activities of DNA-PKcs, thereby hampering the NHEJ repair pathway (8).

ATM and ATR are NHEJ-independent checkpoint regulators which facilitate repair by HR and are inhibited by caffeine (19). Irradiation-induced DNA dsbs activate NHEJ and HR repair pathways and ATM- and ATR-dependent pathways that regulate checkpoint responses. Caffeine inhibits both the ATM- and ATR-regulated checkpoint responses which mainly affect HR (19). Thus, caffeine preferentially inhibits HR repair.

The imprecise NHEJ pathway is the predominant repair process for cells in G0, G1, or early S-phase, and the precise HR pathway is more important for the repair of dsbs in late-S and G2 (1). It was reported that wortmannin sensitized plateau-phase cultured cells, not $\mathrm{P}$ cells, to radiation in vitro (20). Actually, in our study, Q cells showed greater repair capacity than the total cell population in solid tumors with the wild-type p53, and the repair by both total and Q cell populations was more effectively inhibited in combination with wortmannin than caffeine, in spite of an almost isoeffect induced by the drugs in the absence of $\gamma$-ray irradiation (Figs. 2 and 3, Tables I and II). Further, it was also reported that the expression of RAD51, a critical mediator of HR, is repressed by hypoxia in numerous cell lines, irrespective of p53 status $(1,2)$. Taking into account that the intratumor Q cell population includes a much larger hypoxic fraction than the total cell population, as shown in our previous report (18), the repair in Q cells in p53-expressing solid tumors is thought to be predominantly due to the DNA-PK-mediated NHEJ pathway. Anyway, wortmannin may be a promising candidate as a repair inhibitor in radiotherapy for solid tumors with wild-type p53, if its toxicity is within the tolerance level for patients.

Loss-of-function of wild-type TP53 can result in loss of the G1/S cell-cycle checkpoint and an increase in HR $(1,2)$. As p53 seems to interact with RAD51, the absence of normal p53 function is thought to enhance RAD51-mediated DNApairing activity and HR, due to overexpression of RAD51 out of regulation by normal p53 $(1,2)$. Thus, HR is thought to be a principal mechanism of DNA dsb repair in SAS/mp53 cells. The very small repair capacity of SAS/mp53 cells in vivo may show that the repair in solid tumors with a mutant p53 is mainly due to, if anything, the NHEJ rather than HR.

The dose ratios for $\mathrm{SAS} / \mathrm{mp} 53$ cells relative to SAS/neo cells showed that $\mathrm{SAS} / \mathrm{mp} 53$ tumor cells within solid tumors are less radiosensitive than SAS/neo tumor cells. This is consistent with reports that tumor cells with a mutant p53 gene were more radioresistant than those with a wild-type p53 gene (18). Since apparent repair phenomena could be observed in solid tumors with a wild-type p53 gene, the difference in sensitivity between SAS/neo and SAS/mp53 was slightly reduced without significant differences after repair. However, the difference in sensitivity was almost constant with repair-inhibiting wortmannin.

Solid tumors, especially human tumors, are thought to contain a high proportion of Q cells (3). The presence of these cells is probably due, in part, to a microregional deficiency in the concentrations of oxygen, glucose, and other nutritional factors in the tumors caused by poor and heterogeneous tumor vascular supply (3). This deficiency might promote $\mathrm{MN}$ formation in Q tumor cells at 0 Gy (Table I) $(3,21)$. As shown here, Q cells have lower radiosensitivity than $\mathrm{P}$ cells in solid tumors in vivo, irrespective of the p53 status of tumor cells (Table III) (18). This means that more Q cells survive 
after radiotherapy than $\mathrm{P}$ cells. Consequently, the control of $\mathrm{Q}$ cells also has a great impact on the outcome of radiation therapy. Thus, from the viewpoint of the tumor cell-killing effect including intratumor Q-cell control, a treatment modality for enhancing the Q-cell response has to be considered.

\section{Acknowledgements}

This study was supported, in part, by a Grant-in-aid for Scientific Research (C) (18591380) from the Japan Society for the Promotion of Science.

\section{References}

1. Houtgraaf JH, Versmissena $\mathbf{J}$ and van der Giessen WJ: A concise review of DNA damage checkpoints and repair in mammalian cells. Cardiovasc Revascularization Med 7: 165-172, 2006.

2. Horn HF and Vousden KH: Coping with stress: multiple ways to activate p53. Oncogene 26: 1306-1316, 2007.

3. Vaupel P: Tumor microenvironmental physiology and its implications for radiation oncology. Semin Radiat Oncol 14: 197-275, 2004.

4. Masunaga S and Ono K: Significance of the response of quiescent cell populations within solid tumors in cancer therapy. J Radiat Res 43: 11-25, 2002.

5. Hall EL: Repair of radiation damage and the dose-rate effect. In: Radiobiology for the Radiologist. 6th edition. Hall EL (ed.) Lippincott Williams \& Wilkins, Philadelphia, pp60-84, 2006.

6. Karran P: DNA double strand break repair in mammalian cells. Curr Opin Genet Dev 10: 144-150, 2000.

7. Hashimoto M, Rao S, Tokuno O, Yamamoto K, Takaka M, Takeda S and Utsumi H: DNA-PK: the major target for wortmannin-mediated radiosensitization by the inhibition of DSB repair via NHEJ pathway. J Radiat Res 44: 151-159, 2003.

8. Hoekstra MF: Responses to DNA damage and regulation of cell cycle checkpoints by the ATM protein kinase family. Curr Opin Genet Dev 7: 170-175, 1997.

9. Boehm L, Roos WP and Serafin AM: Inhibition of DNA repair by pentoxifyline and related methylxanthine derivatives. Toxicology 193: 153-160, 2003.

10. Ota I, Ohnishi K, Takahashi A, Yane K, Kanata H, Miyahara H, Ohnishi $\mathrm{T}$ and Hosoi $\mathrm{H}$ : Transfection with mutant p53 gene inhibits heat-induced apoptosis in a head and neck cell line of human squamous cell carcinoma. Int J Radiat Oncol Biol Phys 47: 495-501, 2000.
11. Takahashi A: Different inducibility of radiation- or heat-induced p53-dependent apoptosis after acute or chronic irradiation in human cultured squamous cell carcinoma cells. Int J Radiat Biol 77: 215-224, 2001.

12. Jia LQ, Osada M, Ishioka C, Gamo M, Ikawa S, Suzuki T, Shimodaira H, Niitani T, Kudo T and Kuroki T: Screening the p53 status of human cell lines using a yeast functional assay. Mol Carcinog 19: 243-253, 1997.

13. Ohnishi K, Wang X, Takahashi A and Ohnishi T: Contribution of protein kinase $\mathrm{C}$ to $\mathrm{p} 53$ dependent WAF1 induction pathway after heat treatment in human glioblastoma cell lines. Exp Cell Res 238: 399-406, 1998.

14. Franken NA, van Bree $\mathrm{C}$, ten Cate $\mathrm{R}$, van $\mathrm{Oven} \mathrm{CH}$ and Haveman J: Importance of TP53 and RB in the repair of potentially lethal damage and induction of color junctions after exposure to ionizing radiation. Radiat Res 58: 707-714, 2002.

15. Schwartz JL, Razey J, Wiens L, Jordan R and Russel KJ: Functional inactivation of $\mathrm{p} 53$ by HPV-E6 transformation is associated with a reduced expression of radiation-indiced potentially lethal damage. Int J Radiat Biol 75: 285-291, 1999.

16. Kato F, Ootsuyama A, Nomoto S, Kondo S and Norimura T: Threshold effect for teratogenic risk of radiation depends on dose-rate and p53-dependent apoptosis. Int J Radiat Biol 77: 13-19, 2001.

17. Coleman MA, Yin E, Peterson LE, Nelson D, Sorensen K, Tucker JD and Wyrobek AJ: Low-dose irradiation alters the transcript profiles of human lymphoblastoid cells including genes associated with cytogenetic radioadaptive response. Radiat Res 164: 369-382, 2005.

18. Masunaga S, Ono K, Takahashi A, Ohnishi T, Kinashi K and Takagaki M: Radiobiological characteristics of solid tumors depending on the p53 status of the tumor cells, with emphasis on the response of intratumor quiescent cells. Eur J Cancer 38: 718-727, 2002.

19. Wang H, Wang X, Iliakis G and Wang Y: Caffeine could not effectively sensitize homologous recombination repair deficient cells to ionizing radiation induced cell killing. Radiat Res 159: 420-425, 2003.

20. Kubota N, Ozawa F, Okada S, Inada T, Komatsu K and Okatasu R: The phosphatidylinositol 3-kinase inhibitor wortmannin sensitizes quiescent but proliferating MG-63 human osteosarcoma cells to radiation. Cancer Lett 133: 161-167, 1998.

21. Bindra RS, Schaffer PJ, Meng A, Woo J, Maseide K, Roth ME, Lizardi P, Hedley DW, Bristow RG and Glazer PM: Alterations in DNA repair gene expression under hypoxia: elucidating the mechanisms of hypoxia-induced genetic instability. Ann NY Acad Sci 1059: 184-195, 2005. 